\title{
9. Fomento à publicação científica e proteção do conhecimento científico ${ }^{1}$
}

\author{
Financing of the scientific publication and protection of the scientific knowledge
}

Renato Santos de Oliveira Filho² ${ }^{\text {, Bernardo Hochman }}{ }^{3}$, Fabio Xerfan Nahas ${ }^{4}$, Lydia Masako Ferreira ${ }^{5}$

1. Trabalho desenvolvido no Programa de Pós-Graduação em Cirurgia Plástica da Universidade Federal de São Paulo - Escola Paulista de Medicina (UNIFESP - EPM).

2. Professor Adjunto Visitante da Disciplina de Cirurgia Plástica e Professor Orientador do Programa de Pós-Graduação em Cirurgia Plástica da UNIFESP - EPM.

3. Mestre em Medicina e pós-graduando em nível de doutorado pelo Programa de Pós-Graduação em Cirurgia Plástica da UNIFESP - EPM.

4. Professor Adjunto Visitante da Disciplina de Cirurgia Plástica e Professor Orientador do Programa de Pós-Graduação em Cirurgia Plástica da UNIFESP - EPM.

5. Professora Titular da Disciplina de Cirurgia Plástica e Coordenadora do Programa de Pós-Graduação em Cirurgia Plástica da UNIFESP - EPM.

\section{RESUMO}

Fomento à publicação científica e proteção do conhecimento científico ${ }^{1}$

O trabalho científico atinge sua finalidade maior através de sua publicação. É indiscutível a importância atual das agências de fomento à pesquisa para que se possa desenvolver, finalizar e publicar os trabalhos científicos. As principais agências de fomento abordadas neste artigo são: a Coordenação de Aperfeiçoamento de Pessoal de Nível Superior (CAPES), o Conselho Nacional de Desenvolvimento Científico e Tecnológico (CNPq) e a Fundação de Amparo à Pesquisa do Estado de São Paulo (FAPESP). As atividades da CAPES podem ser agrupadas em quatro grandes linhas de ação: a) avaliação da pós-graduação stricto sensu; b) acesso e divulgação da produção científica; c) investimentos na formação de recursos de alto nível no país e exterior e d) promoção da cooperação científica internacional. Embora não haja uma linha direta de apoio à publicação científica, praticamente todas as ações da CAPES acabam por contribuir para a concretização de trabalhos científicos e suas publicações. O CNPq tem duas atividades básicas: o fomento à pesquisa e a formação de recursos humanos. Disponibiliza aos pesquisadores auxílio à divulgação e publicação científicas. O auxílio à editoração tem como objetivo apoiar publicações técnico-científicas nacionais, mantidas e editadas por instituição ou sociedade científica brasileira de âmbito nacional. Através do auxílio à promoção de eventos científicos, o CNPq apóia realização no país de congressos, simpósios e outros eventos similares de curta duração. O CNPq disponibiliza a Plataforma Lattes através da qual é possível preencher e acessar o Curriculum Lattes, hoje indispensável aos pesquisadores. A FAPESP financia publicação de periódicos, artigos e livros que exponham resultados originais de pesquisa realizada por pesquisador do Estado de São Paulo. Financia parcialmente a participação de pesquisadores em reuniões científicas ou tecnológicas, no Brasil ou no exterior, para apresentação de trabalho de sua autoria, não publicado, que exponha resultados inéditos. $\mathrm{O}$ Brasil vem aumentando sua participação nas publicações científicas internacionais. A nova realidade exige, além da publicação, uma preocupação com a proteção do conhecimento. A mudança da cultura da publicação para a da publicação com proteção do conhecimento é recente no país, tanto por parte das empresas, dos institutos de pesquisa, das universidades como das agências de fomento à pesquisa. Os pesquisadores devem-se conscientizar da necessidade de proteção do conhecimento e as agências de fomento considerar, como critérios de avaliação de produtividade científica, um peso mais equilibrado entre as publicações científicas, os resultados patenteáveis e o know how.

Descritores: Projetos de Pesquisa. Financiamento Governamental. Doações. Financiamento da Saúde. Patentes. Propriedade Intelectual.

\begin{abstract}
The main purpose of a study is its publication on a scientific journal. Research financing agencies are important institutions so that studies can be developed and published. The most important research financing agencies that are discussed in this article are: “Coordenação de Aperfeiçoamento de Pessoal de Nível Superior” (CAPES), “Conselho Nacional de Desenvolvimento Científico e Tecnológico” (CNPq) and "Fundação de Amparo à Pesquisa do Estado de São Paulo” (FAPESP). CAPES' activities can be grouped in four different strategy lines: a) it evaluates the stricto sensu, at the postgraduation level; b) it provides access and development of scientific research; c) it provides investment on the development of high qualified human resources in Brazil and abroad, and d) it promotes international scientific cooperation. Although CAPES does not support directly scientific publications, almost all actions of this agency contribute to the development of scientific research and publication. $\mathrm{CNPq}$ has two main purposes: financing researches and development of human resources. It provides the researchers with financial aid to scientific publication. The grants for editing were specifically created for supporting the national scientific and technical publications edited by Brazilians institutions or societies. CNPq can also support Congresses, Symposiums and similar short-term courses. The Plataforma Lattes is also a branch of CNPq on which the Curriculum Lattes is available. This site has the curriculum vitae of the scientific community and
\end{abstract}


is of great value for researchers. FAPESP also finances journal publications, articles and books that bring up original results of studies made by researchers from the state of São Paulo. It finances, partially, the travel expenses of innovative papers authors in meetings within the country or abroad. Brazilian authors are increasing the number of international publications. Universities, research institutes, financing agencies and private companies are more and more concerned with knowledge property. Researchers must understand the need of knowledge property and the financing agencies have to consider the patents achieved as a criteria of evaluation of scientific production.

Key words: Research Design. Financing, Government. Gift Giving. Financing, Health. Patents. Intellectual Property.

\section{Introdução}

O trabalho científico atinge sua finalidade maior através de sua publicação. A apresentação de trabalhos em congressos, simpósios ou em outros encontros científicos é importante, mas deve ser entendida como uma etapa anterior à sua publicação. É uma oportunidade que o pesquisador tem para ouvir críticas, sugestões e aperfeiçoar suas idéias. A realização de trabalhos de iniciação científica por alunos de graduação, sob orientação de um pesquisador, tem tido estímulos crescentes pelas universidades e agências de fomento à pesquisa. O desenvolvimento de pesquisas dentro de um programa de pós-graduação precisa, na maioria das vezes, de apoio financeiro. É indiscutível a importância atual das agências de fomento à pesquisa para que se possa desenvolver, finalizar e publicar os trabalhos científicos. As principais agências de fomento abordadas neste artigo são a Coordenação de Aperfeiçoamento de Pessoal de Nível Superior (CAPES), o Conselho Nacional de Desenvolvimento Científico e Tecnológico (CNPq) e a Fundação de Amparo à Pesquisa do Estado de São Paulo (FAPESP).

\section{CAPES}

A CAPES vem desempenhando papel fundamental na expansão e consolidação da pós-graduação stricto sensu (mestrado e doutorado) em todos os estados da Federação representando uma grande conquista no campo da Educação e da Ciência e Tecnologia. Foi criada em 1951 com o objetivo de "assegurar a existência de pessoal especializado em quantidade e qualidade suficientes para atender às necessidades dos empreendimentos públicos e privados que visam ao desenvolvimento do país”. ${ }^{1}$

As atividades da CAPES podem ser agrupadas em quatro grandes linhas de ação, cada qual desenvolvida por um conjunto estruturado de programas: a) avaliação da pós-graduação stricto sensu; b) acesso e divulgação da produção científica; c) investimentos na formação de recursos de alto nível no país e exterior; d) promoção da cooperação científica internacional. Os resultados da avaliação da pós-graduação servem de base para a formulação de políticas para a área de pós-graduação, bem como para o dimensionamento das ações de fomento - bolsas de estudo, auxílios, apoios -, estabelecendo, ainda, critérios para o reconhecimento pelo Ministério da Educação dos cursos de mestrado e doutorado novos e em funcionamento no Brasil. A CAPES promove o acesso e divulgação da produção científica através do Portal Periódicos, do acesso à busca e consulta a informações sobre teses e dissertações defendidas junto aos programas de pós-graduação do país e através do INFOCAPES que sucedeu à Revista Brasileira de Pós-Graduação em 2004. O Programa de Apoio a Eventos no País - PAEP, concede recursos a eventos de caráter científico, tecnológico e cultural de curta duração, promovidos por associações e sociedades nacionais científicas, de pós-graduação e de pesquisa com abrangência nacional e internacional. Os eventos apoiados devem apresentar interesse inequívoco para a pós-graduação stricto sensu. Embora não haja uma linha direta de apoio à publicação científica, praticamente todas as ações da CAPES acabam por contribuir para a concretização de trabalhos científicos e suas publicações. O relacionamento do pesquisador com a CAPES é feito por meio do site www.capes.gov.br.

\section{CNPq}

Os investimentos feitos pelo CNPq, órgão vinculado ao Ministério da Ciência e Tecnologia, são direcionados para a formação e absorção de recursos humanos e financiamento de projetos de pesquisa que contribuem para o aumento da produção de conhecimento e geração de novas oportunidades de crescimento para o país. Tem duas atividades básicas: o fomento à pesquisa e a formação de recursos humanos. O CNPq disponibiliza aos pesquisadores auxílio à divulgação e publicação científicas, além de outros benefícios. ${ }^{2}$

\section{Auxílio à editoração}

Tem como objetivo apoiar publicações técnicocientíficas nacionais, mantidas e editadas por instituição ou sociedade científica brasileira de âmbito nacional, contribuindo para elevar o nível de qualidade, forma e conteúdo dos periódicos para divulgação no Brasil e exterior. Os requisitos para a publicação são os seguintes:

1. publicar mais de $50 \%$ de artigos de natureza científica e/ou tecnológica, apresentando resultados originais e inéditos, de uma determinada área do conhecimento.

2. Ter abrangência nacional quanto a colaboradores, corpo editorial e conselho científico (referees), conforme norma específica do programa editorial.

3. Apresentar o Número Internacional Normalizado para Publicações Seriadas (ISSN), obtido através do IBICT/CNPq.

Os documentos indispensáveis para inscrição são: 


\section{Formulário Eletrônico de Propostas.}

2. Preenchimento/atualização do Currículo Lattes pelo solicitante.

3. Orçamento detalhado de duas gráficas, com indicação da que foi escolhida e justificativa da opção.

4. Um exemplar dos dois últimos números publicados.

5. Número Internacional Normalizado para Publicações Seriadas (ISSN), obtido através do IBICT/CNPq.

\section{Auxílio para participação em eventos científicos}

Apóia a participação de pesquisador em eventos científicos no exterior, tais como congressos e similares, para apresentação de trabalhos ou intercâmbio científico e/ou tecnológico. Incentiva também estágios, visitas e cursos de curta duração, para aquisição de conhecimentos específicos e necessários ao desenvolvimento da pesquisa científica e/ou tecnológica, por meio do aperfeiçoamento, reciclagem ou treinamento. Os requisitos e condições para participação em congressos e similares são os seguintes:

1. Possuir o título de doutor ou formação equivalente.

2. Ter qualificação, experiência e desempenho inequívoco em sua área de atuação.

3. Apresentar programação do evento e/ou cartaconvite.

4. Apresentar cópia do texto completo (versão final) do trabalho/artigo a ser apresentado, quando pertinente.

Ainda, requere-se demonstrar participação destacada na seguinte ordem de prioridade:

1. Conferencista convidado.

2. Debatedor convidado e/ou presidente em sessões de eventos.

3. Palestrante convidado para apresentação completa do trabalho em sessão regular ou subsessão do evento.

4. Participante com apresentação de trabalho aceito pela organização do evento, sendo, nesse caso, exigido interstício de dois anos para novas concessões.

\section{Auxílio à promoção de Eventos Científicos}

Apóia a realização no país de congressos, simpósios, workshops, seminários, ciclos de conferências, cursos e outros eventos similares de curta duração e relacionados à ciência e/ou tecnologia. Requere-se que o solicitante seja pesquisador de comprovada qualificação e experiência, ou que seja dirigente de associação científica e/ou tecnológica, de âmbito nacional, assim como que apresente o programa do evento e com orçamento detalhado.

\section{Plataforma Lattes}

Conjunto de sistemas computacionais do CNPq que visa a compatibilizar e integrar as informações em toda interação da Agência com seus usuários. Seu objetivo maior é aprimorar a qualidade dessas informações e racionalizar o trabalho dos pesquisadores e estudantes no seu preenchimento. Nesta plataforma é possível preencher e acessar o Curriculum Lattes, hoje indispensável aos pesquisadores.

\section{Programa Institucional de Bolsas de Iniciação Científica}

O PIBIC desperta a vocação científica e incentiva novos talentos potenciais entre estudantes de graduação, mediante sua participação em projetos de pesquisa, preparando-os para o ingresso na pósgraduação. Certamente, o PIBIC está contribuindo para a redução do tempo médio de titulação de mestres e doutores. O acesso e contatos com o CNPq devem ser feito pelo site http://www.cnpq.br.

\section{FAPESP}

As Fundações de Amparo à Pesquisa (“FAPs”) são instituições estaduais que dão suporte e fomento à pesquisa em seus estados. A FAPESP é uma das principais agências de fomento à pesquisa científica e tecnológica do país. Está ligada à Secretaria de Ciência, Tecnologia, Desenvolvimento Econômico e Turismo. ${ }^{3}$ Bolsas e Auxílios são os meios tradicionais oferecidos pela FAPESP para o fomento da pesquisa científica e tecnológica em todas as áreas do conhecimento: Ciências Biológicas, Ciências da Saúde, Ciências Exatas e da Terra, Engenharias, Ciências Agrárias, Ciências Sociais Aplicadas, Ciências Humanas, Lingüística e Letras e Artes. As Bolsas se destinam a estudantes de graduação e pós-graduação de instituições de ensino e pesquisa paulistas. Os Auxílios se destinam aos pesquisadores de instituição de ensino e pesquisa do Estado de São Paulo com titulação mínima de doutor. As Bolsas e Auxílios são concedidos dentro de três linhas de financiamento: Linhas Regulares, Programas Especiais e Inovação Tecnológica. As Linhas Regulares estão voltadas para o atendimento da demanda espontânea (a chamada demanda de balcão) dos pesquisadores ligados às universidades e institutos de pesquisa sediados no Estado de São Paulo. Constituem, portanto, um sólido suporte das propostas de pesquisa livremente pensadas e formuladas pela comunidade científica e tecnológica paulista. Os Programas Especiais voltam-se para a superação de carências existentes (ou até mesmo antevistas) no Sistema de Ciência e Tecnologia do Estado. Já a linha de Inovação Tecnológica compreende diversos programas 
cujas pesquisas têm grande potencial de desenvolvimento de novas tecnologias e de aplicação prática em diversas áreas do conhecimento. Os programas dessas duas linhas, financiados sobretudo com receitas patrimoniais da instituição, são os pilares da ação indutora, orientadora, do desenvolvimento científico e tecnológico que também cabe à FAPESP desempenhar, em afinação com a política de Ciência e Tecnologia do governo estadual. Candidatos a apoio da FAPESP, dentro das Linhas Regulares ou nos Programas Especiais, dispõem de formulários apropriados a cada caso para encaminhar suas propostas. Há também alguns procedimentos a serem observados pelo solicitante de apoio da Fundação. No processo de avaliação todas as propostas encaminhadas à FAPESP, enquadradas em quaisquer de seus programas, são avaliadas quanto ao mérito científico ou tecnológico e quanto à sua adequação às normas e critérios de prioridade da FAPESP. Essa avaliação é feita por pares, assessores escolhidos entre cientistas de reconhecida competência, de acordo com a natureza e a área do conhecimento em que se insere cada projeto. A FAPESP tem, assim, uma rede de mais de 6 mil assessores voluntários, a maioria pesquisadores em atividade no Estado de São Paulo, enquanto algumas centenas estão espalhados pelo Brasil e Exterior.

\section{Auxílio à publicação}

Destina-se ao financiamento parcial e proporcional de publicação de periódicos, artigos e livros que exponham resultados originais de pesquisa realizada por pesquisador do Estado de São Paulo. Como requisitos do pesquisador, o mesmo deve ter título de doutor ou equivalente e ter vínculo com instituição de pesquisa do Estado de São Paulo.

\section{Participação em reunião científica e/ou tecnológica}

Destina-se a financiar parcialmente a participação de pesquisadores do Estado de São Paulo em reuniões científicas ou tecnológicas, no Brasil ou no exterior, para apresentação de trabalho de sua autoria, não publicado, resultado de projeto de pesquisa financiado pela FAPESP, que exponha resultados inéditos de pesquisa. A apresentação de trabalho de pesquisa científica e/ou tecnológica, não resultante de apoio da FAPESP, poderá ser financiada apenas em condições excepcionais. Serão avaliados a importância do evento, a modalidade de apresentação, a regularidade da produção divulgada em publicações e o conjunto de solicitações referentes ao mesmo evento, bem como a relação do trabalho a ser apresentado com projetos de pesquisa financiados pela FAPESP. O pesquisador solicitante deve ter título de doutor ou qualificação equivalente, ter expressiva produção científica ou tecnológica e proficiência no idioma da reunião.

\section{Publicação e proteção do conhecimento}

Os cientistas e inventores precisam dispor de proteção para os aplicativos inovadores que são frutos de seu trabalho árduo e de sua imaginação. Nossas universidades públicas ainda estão presas ao princípio da publicação. O esforço individual e institucional agrega conhecimento à ciência. O resultado é que esse conhecimento se realmente dotado de alguma praticidade, acabará nas mãos de outros que dele se beneficiarão. ${ }^{4}$

A publicação é essencial e o Brasil vem aumentando sua participação nas publicações científicas internacionais. Todavia, ainda que atual, oportuno e necessário, publicar não é suficiente, pelo menos na área tecnológica. Devemos considerar a nova realidade em que vivemos, promovida pelos avanços tecnológicos da comunicação, especialmente a internet. Essa nova realidade tem repercussões sobre a Universidade e sobre suas políticas de ciência e tecnologia e sobre as políticas de publicações. Estamos entrando irreversivelmente numa nova ordem mundial, que tem como denominador comum e diferenciador, o conhecimento. Isto significa, concretamente, que além de publicar, faz-se necessário assegurar a devida proteção ao conhecimento gerado por nossos pesquisadores. Do contrário, o que renunciarmos em nome de um idealismo, acabará inapelavelmente sendo apropriado por outros, sintonizados com a nova ordem dos fatos. Em suma, está-se dizendo que não é razoável se pensar hoje, na era do conhecimento, da mesma forma que na era anterior, que está passando, rapidamente. A temática da proteção do conhecimento e transferência de tecnologias é complexa. Deve-se superar os pré-conceitos e a desinformação e a questão da proteção do conhecimento ganhará grande amplitude no âmbito das instituições de pesquisa e das universidades. Essas, surgidas com a missão de gerar conhecimento científico e formar recursos humanos qualificados para a sociedade, progressivamente, são induzidas a participar de atividades que têm como foco a promoção do desenvolvimento econômico, ou simplesmente, a geração de conhecimentos tecnológicos capazes de gerar valor econômico aos produtos e serviços. O lema "publicar ou perecer", muito comum nas universidades americanas e com variantes em todo o mundo, está mudando, sem perder seu imperativo, que continua válido. Essa máxima reflete o espírito que pressionou gerações de pesquisadores à produção intelectual. Essa cultura, todavia, começou a mudar e, hoje, em muitos ambientes acadêmicos, é conjugada com outra, que pode ser resumida na necessidade crescente de proteção do conhecimento. Aos poucos, particularmente nas áreas tecnológicas, ganha relevância crescente uma nova realidade: antes de publicar, proteger. A passagem da cultura da publicação para a da publicação com proteção do conhecimento é recente no país, tanto por parte das empresas, como por parte dos institutos de pesquisa, das universidades e, por incrível que possa parecer, das agências de fomento à pesquisa. Sendo assim, é preciso conscientizar os pesquisadores quanto à necessidade de proteção do conhecimento, sensibilizar as agências de fomento para que considerem como critérios de avaliação de produtividade científica, um peso mais equilibrado entre as publicações científicas, os resultados patenteáveis e o know how. Os frutos, logicamente, 
devem ser compartilhados entre o financiador e o financiado.

\section{Referências}

1. Capes.gov [homepage on the Internet]. Brasilia: Coordenação de Aperfeiçoamento de Pessoal de Nível Superior; [updated 2005 Sep 4; cited 2005 Sep 4]. Available from: http://www.capes.gov.br/capes/portal.

2. CNPq.org [homepage on the Internet]. Brasilia: Conselho Nacional de Desenvolvimento Científico e
Tecnológico; [updated 2005 Sep 4; cited 2005 Sep 4]. Available from: http//www.cnpq.br.

3. FAPESP.org [homepage on the Internet]. Sao Paulo: Fundação de Amparo à Pesquisa do Estado de São Paulo; [updated 2005 Sep 4; cited 2005 Sep 4]. Available from: URL:http//www.fapesp.br.

4. Sócio Estatística Pesquisa \& Consultoria Ltda. [homepage on the Internet]. Itabuna; [cited 2005 Sep 4]. Publicação e proteção do conhecimento; [about 17 screens]. Available from:

http://www.socioestatistica.com.br/publicacao.htm.

\section{Correspondência:}

Renato Santos de Oliveira Filho

Disciplina de Cirurgia Plástica / Departamento de Cirurgia

Universidade Federal de São Paulo (UNIFESP - EPM)

Rua Napoleão de Barros, 715 - $4^{\circ}$ andar

04024-900 São Paulo-SP Brasil

Tel: (5511) 5576-4118

renato.dcir@epm.br

\section{Como citar este artigo:}

Oliveira Filho RS, Hochman B, Nahas FX, Ferreira LM. Fomento à publicação científica e proteção do conhecimento científico. Acta Cir Bras [periódico na Internet] 2005;20 Suppl. 2:35-9. Disponível em URL: http://www.scielo.br/acb 